\title{
On a new linear operator formulated by Airy functions in the open unit disk
}

\author{
Rabha W. Ibrahim ${ }^{1 *}$ (D) and Dumitru Baleanu $2,3,4$
}

\section{"Correspondence:}

rabhaibrahim@yahoo.com

'IEEE: 94086547, Kuala Lumpur,

59200, Malaysia

Full list of author information is

available at the end of the article

\begin{abstract}
In this note, we formulate a new linear operator given by Airy functions of the first type in a complex domain. We aim to study the operator in view of geometric function theory based on the subordination and superordination concepts. The new operator is suggested to define a class of normalized functions (the class of univalent functions) calling the Airy difference formula. As a result, the suggested difference formula joining the linear operator is modified to different classes of analytic functions in the open unit disk.
\end{abstract}

Keywords: Analytic function; Univalent function; Subordination; Open unit disk

\section{Introduction}

The field of geometric function theory is rich with different types of linear, differential, integral, and mixed operators. A few linear operators have been formulated in this field, such as the Carlson-Shaffer operator [1], hypergeometric linear operator [2, 3], and FoxWrite linear operator [4]. In this note, we present a linear operator formulated by the Airy functions [5], which are special functions determined by the hypergeometric function of a complex variable. These functions are solutions for the Airy equation $f^{\prime \prime}(z)-z f(z)=0$. The class of these differential equations plays an important role in applied sciences such as optics, economy, and astronomy. The greatest benefit of Airy functions in mathematical studies is development in the fields of special functions and statistical studies [6]. The formula of the Airy function of a complex variable is given by

$$
\begin{aligned}
A l(z) & =\frac{1}{2 \pi i} \int_{U} \exp \left(\frac{\zeta^{3}}{3}-z \zeta\right) d \zeta \\
& =\frac{1}{\pi 3^{2 / 3}} \sum_{n=0}^{\infty} \frac{\Gamma\left(\frac{n+1}{3}\right)}{n !} \sin \left(\frac{2 \pi(n+1)}{3}\right)\left(3^{1 / 3} z\right)^{n} \\
& =\frac{1}{\pi 3^{2 / 3}} \sum_{n=0}^{\infty} \frac{\Gamma\left(\frac{n+1}{3}\right)}{n !}\left(\frac{\pi}{\Gamma\left(1-\frac{2(n+1)}{3}\right) \Gamma\left(\frac{2(n+1)}{3}\right)}\right)\left(3^{1 / 3} z\right)^{n}
\end{aligned}
$$

(c) The Author(s) 2021. This article is licensed under a Creative Commons Attribution 4.0 International License, which permits use, sharing, adaptation, distribution and reproduction in any medium or format, as long as you give appropriate credit to the original author(s) and the source, provide a link to the Creative Commons licence, and indicate if changes were made. The images or other third party material in this article are included in the article's Creative Commons licence, unless indicated otherwise in a credit line to the material. If material is not included in the article's Creative Commons licence and your intended use is not permitted by statutory regulation or exceeds the permitted use, you will need to obtain permission directly from the copyright holder. To view a copy of this licence, visit http://creativecommons.org/licenses/by/4.0/. 


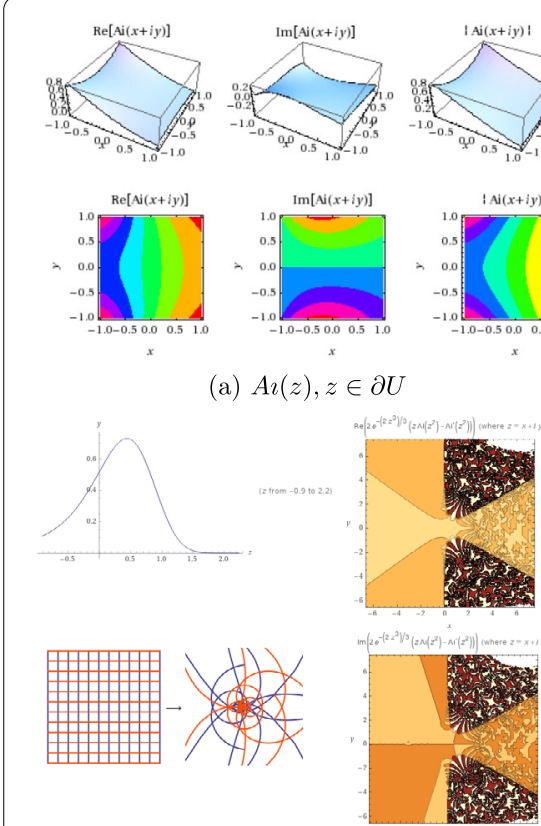

(c) Airy distribution function

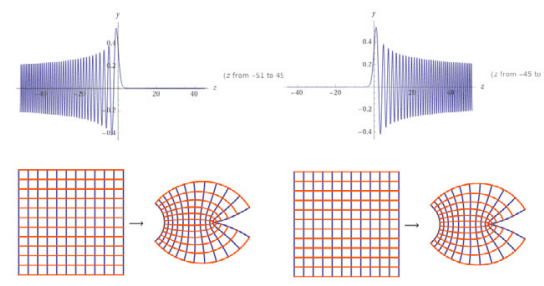

(b) $A \imath(z)$ - chaotic behavior

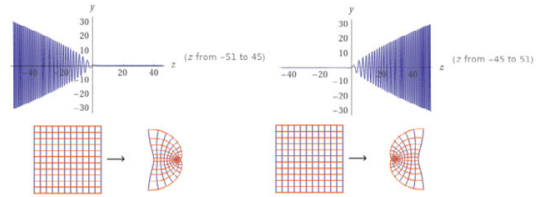

(d) The modified Airy function $\mathbb{A} \imath$

Figure 1 Three behavior graphs of Airy $\operatorname{Al}(z)$ and Airy distribution functions respectively

$$
\begin{aligned}
& \approx \sum_{n=0}^{\infty}\left(\frac{\Gamma(3 / 2)}{\Gamma(1 / 2)}\right)\left(\frac{\Gamma\left(\frac{n+1}{3}\right)}{\Gamma\left(\frac{1-2 n)}{3}\right) \Gamma\left(\frac{2(n+1)}{3}\right)}\right)\left(\frac{1}{\Gamma\left(1+\frac{n}{3}\right)}\right) \frac{z^{n}}{n !} \\
& ={ }_{2} \Psi_{4}\left[\begin{array}{cccc}
(3 / 2,0) & (1 / 3,1 / 3) \\
(1 / 2,0) & (1 / 3,-2 / 3) & (2 / 3,2 / 3) & (1,1 / 3)
\end{array} ; z\right],
\end{aligned}
$$

where the integral is over the open unit disk $U:=\{z \in \mathbb{C}:|z|<1\}, 3^{n / 3} \approx \frac{1}{\Gamma\left(1+\frac{n}{3}\right)}, \frac{1}{3^{2 / 3}} \approx$ $\frac{\Gamma(3 / 2)}{\Gamma(1 / 2)}$, and ${ }_{p} \Psi_{q}$ is the Fox-Wright function having the series

$$
{ }_{p} \Psi_{q}\left[\begin{array}{cccc}
\left(a_{1}, \aleph_{1}\right) & \left(a_{2}, \aleph_{2}\right) & \cdots & \left(a_{p}, \aleph_{p}\right) \\
\left(b_{1}, B_{1}\right) & \left(b_{2}, B_{2}\right) & \cdots & \left(b_{q}, B_{q}\right)
\end{array}\right]=\sum_{n=0}^{\infty} \frac{\Gamma\left(a_{1}+\aleph_{1} n\right) \cdots \Gamma\left(a_{p}+\aleph_{p} n\right)}{\Gamma\left(b_{1}+B_{1} n\right) \cdots \Gamma\left(b_{q}+B_{q} n\right)} \frac{z^{n}}{n !} .
$$

Moreover, the Airy distribution function of the random variable $\chi$ is given by the formula (see Fig. 1)

$$
P(\chi)=2 e^{-2 / 3 \chi^{3}}\left[\chi A l\left(\chi^{2}\right)-(A l)^{\prime}\left(\chi^{2}\right)\right] .
$$

By using the complex probability [7, 8], Eq. (2) can be extended to the complex domain as follows:

$$
P(z)=2 e^{-2 / 3 z^{3}}\left[\chi A l\left(z^{2}\right)-(A \iota)^{\prime}\left(z^{2}\right)\right] .
$$




\section{Methods}

Let $\Lambda$ be the class of normalized functions in $U$ having the series

$$
f(z)=z+\sum_{n=2}^{\infty} \varphi_{n} z^{n}, \quad z \in U .
$$

And let $\mathbb{S}^{*}, \mathbb{C}$ be the classes of starlike and convex functions respectively. The Hadamard product (convolution product) is defined by the series

$$
f(z) * g(z)=z+\sum_{n=2}^{\infty} \varphi_{n} \psi_{n} z^{n}, \quad z \in U,
$$

where $g(z)=z+\sum_{n=2}^{\infty} \psi_{n} z^{n}$. An analytic function $f \in U$ is on subordination with the analytic function $g \in U$ represented by $f \prec g$ if there occurs an analytic function $w$ with $|w(z)| \leq|z|$ such that $f=(g(w))$. In the sequel, we shall use the class of normalized functions $\Lambda$ satisfying $f(0)=0$ and $f^{\prime}(0)=1$ having the series (see [9])

$$
f(z)=z+\sum_{n=2}^{\infty} \phi_{n} z^{n}, \quad z \in U
$$

Moreover, two analytic functions $f$ and $g$ in $U$, the function $f$ is majored by $g(f \ll g)$ if there is an analytic function $\varpi,|\varpi|<1$ such that $f(z)=\varpi(z) g(z)$. Note that there is a connection between majorization and subordination concepts (see [10,11]). Under some conditions, we have $f \ll g \Leftrightarrow f \prec g$.

\subsection{Linear operator}

We shall use the Hadamard product to define the new linear operator using the Airy function of a complex variable $z \in U$. Construct the modified Airy function as follows:

$$
\begin{aligned}
\mathbb{A}_{l}(z) & :=\left(3^{2 / 3} \Gamma(2 / 3) z\right) A_{l}(z) \\
& =z-\left(\frac{3^{1 / 3} \Gamma(2 / 3)}{\Gamma(1 / 3)}\right) z^{2}+\cdots+O\left(z^{6}\right) .
\end{aligned}
$$

Define a linear operator $\Delta: \Lambda \rightarrow \Lambda$ as follows:

$$
\begin{aligned}
\Delta f(z) & =\mathbb{A} l(z) * f(z) \\
& =z+\sum_{n=2}^{\infty} \delta_{n} \varphi_{n} z^{n} \in \Lambda,
\end{aligned}
$$

where $\delta_{n}$ indicates the coefficient of $\mathbb{A} l(z)$. The linear operator (6) is called the Airy linear operator of normalized analytic functions. It is well known that for $\Re(z)>0$ the Airy function is convex with $\Re\left(A_{l}(z)\right)>0$. We have the following proposition, which indicates that the linear operator can be formulated by a set of special functions and other properties, which are easily proved. Therefore, we omit the proof.

Proposition 1 Consider the linear operator $\Delta f(z), f \in \Lambda$. Then it can be formulated by the following special functions: 


$$
\Delta f(z)=\left(\frac{z A l(z) G(5 / 3) 3^{2 / 3}}{G(2 / 3)}\right) * f(z)
$$

$$
\Delta f(z)=\left(\frac{z G(5 / 3) 3^{2 / 3}\left(I_{-1 / 3}\left(\frac{2 z^{3 / 2}}{3}\right) \sqrt{z}-\frac{z I_{1 / 3}\left(\frac{2 z^{3 / 2}}{3}\right)}{\sqrt{z}}\right)}{3 G(2 / 3)}\right) * f(z)
$$

$$
\Delta f(z)=\left(\frac{z G(5 / 3) 3^{2 / 3}\left(J_{-1 / 3}\left(\frac{2}{3} i z^{3 / 2}\right) \sqrt{z e^{i \pi / 3}}-\frac{z J_{1 / 3}\left(\frac{2}{3} i z^{3 / 2}\right)}{\sqrt{z e^{i \pi / 3}}}\right)}{(3 G(2 / 3))}\right) * f(z)
$$

$$
\Delta f(z)=\frac{3^{\frac{1}{4}} \pi^{\frac{2}{3}}\left(\frac{(2+\sqrt{3})\left(1+\frac{2(\sqrt{2}+\sqrt{3})(5+3 \sqrt{3})}{(3+\sqrt{2}+\sqrt{3})^{2}}\right)}{\left(2+\frac{1}{\sqrt{2+\sqrt{3}}}\right) K\left(\frac{\left(1-\frac{(2(\sqrt{2}+\sqrt{3})(5+3 \sqrt{3}))}{(3+\sqrt{2}+\sqrt{3})^{2}}\right)^{2}}{\left(1+\frac{2(\sqrt{2}+\sqrt{3})(5+3 \sqrt{3})}{(3+\sqrt{2}+\sqrt{3})^{2}}\right)^{2}}\right)}\right)^{1 / 3} z A i(z)}{2^{\frac{4}{9}}} * f(z) ;
$$

$$
(\mathbb{A} \iota(z))^{\prime}=3^{2 / 3} \Gamma\left(\frac{2}{3}\right)\left(A \imath(z)+z A \iota^{\prime}(z)\right)
$$

$$
\Re(\mathbb{A} l(z)) \approx 0.385116, \quad \Re(z)=0.88405
$$

$$
\int_{U} \mathbb{A}_{l}(z) d z=3^{2 / 3} \Gamma(2 / 3) A i^{\prime}(z), \quad\left|\int_{U} \mathbb{A}_{l}(z) d z\right| \approx 0.419648
$$

where $G(\omega)$ is the Barnes G-function, $I_{n}(\omega)$ is the modified Bessel function of the first kind, $J_{n}(\omega)$ is the Bessel function of the first kind, and $K$ is the complete elliptic integral of the first kind.

\subsection{The difference formula}

We proceed to defining our class of normalized analytic functions based on the Airy equation. The Airy equation can be reformulated by the structure

$$
\frac{z f^{\prime \prime}(z)}{f^{\prime}(z)}-\frac{z^{3}}{\frac{z f^{\prime}(z)}{f(z)}}=0, \quad f \in \Lambda
$$

Our structure of the class of analytic functions is given by the Airy difference formula

$$
\Psi(z):=\frac{z f^{\prime \prime}(z)}{f^{\prime}(z)}-\frac{z^{3}}{\frac{z f^{\prime}(z)}{f(z)}} .
$$

By utilizing the linear operator $\Delta f$, we have the following class. 
Definition 1 Let $f \in \Lambda$. Define the class of analytic functions $\Lambda t_{s}$ satisfying the following subordination:

$$
\begin{gathered}
\Psi_{s}(z):=\frac{1}{2 s}\left(\frac{z f^{\prime \prime}(z)}{f^{\prime}(z)}-\frac{z^{3}}{\frac{z f^{\prime}(z)}{f(z)}}\right) \prec \Delta f(z) \\
(z \in U, f \in \Lambda, s \in \mathbb{R} \backslash\{0\}) .
\end{gathered}
$$

Example 1 Let $f(z)=\frac{z}{(1-z)^{s}}$, we have the formula

$$
\begin{aligned}
\frac{1}{2 s}\left(\frac{z f^{\prime \prime}(z)}{f^{\prime}(z)}-\frac{z^{3}}{\left.\frac{z f^{\prime}(z)}{f(z)}\right)=}\right. & \frac{\frac{s z((s-1) z+2)}{(1-z)((s-1) z+1)}-\frac{(1-z) z^{3}}{(s-1) z+1}}{2 s} \\
= & z+\frac{1}{2}(3-s) z^{2}+\frac{\left(s^{3}-3 s^{2}+4 s-1\right) z^{3}}{(2 s)}+\left(-s^{3} / 2+2 s^{2}-3 s+3\right) z^{4} \\
& +\frac{1}{2}\left(s^{4}-5 s^{3}+10 s^{2}-11 s+7\right) z^{5}+O\left(z^{6}\right) .
\end{aligned}
$$

Moreover, we have

$$
\begin{aligned}
\Delta f(z)= & \left(z-\frac{3^{1 / 3} z^{2} \Gamma(2 / 3)}{\Gamma(1 / 3)}+\frac{z^{4}}{6}-\frac{z^{5} \Gamma(2 / 3)}{4\left(3^{2 / 3} \Gamma(1 / 3)\right)}+O\left(z^{6}\right)\right) \\
& *\left(z+s z^{2}+\frac{1}{2 !} s(s+1) z^{3}+\frac{1}{3 !} s(s+1)(s+2) z^{4}\right. \\
& \left.+\frac{1}{4 !} s(s+1)(s+2)(s+3) z^{5}+O\left(z^{6}\right)\right) \\
= & z-\frac{3^{1 / 3} s z^{2} \Gamma(2 / 3)}{\Gamma(1 / 3)}+\frac{1}{3 !} s(s+1)(s+2) \frac{z^{4}}{6} \\
& -\left(\frac{1}{4 !} s(s+1)(s+2)(s+3)\right) \frac{z^{5} \Gamma(2 / 3)}{4\left(3^{2 / 3} \Gamma(1 / 3)\right)}+O\left(z^{6}\right) .
\end{aligned}
$$

It is clear that the formula

$$
\left.\Psi_{s}(z)\right|_{z=0}=\left.\frac{1}{2 s}\left(\frac{z f^{\prime \prime}(z)}{f^{\prime}(z)}-\frac{z^{3}}{\frac{z f^{\prime}(z)}{f(z)}}\right)\right|_{z=0}=\left.\Delta f(z)\right|_{z=0}=0 .
$$

And by comparing the coefficients of $\Delta f(z)$ and $\Psi_{s}(z)$, we have that the unique real root of $s^{3}-3 s^{2}+4 s-1=0$ is

$$
s=1-\left(\frac{2}{3(-9+\sqrt{93})}\right)^{1 / 3}+\frac{\left(\frac{1}{2}(-9+\sqrt{93})\right)^{1 / 3}}{3^{2 / 3}} \approx 0.31767 .
$$

As a conclusion, we have

$$
\Psi_{s}(z) \ll \Delta\left(\frac{z}{(1-z)^{s}}\right), \quad s \leq 0.31767, z \in U .
$$

Note that the function $\frac{z}{(1-z)^{s}}$ is called the generalized Koebe function, which is an extreme function in $U$ for some values of $s$. 
Our investigation is based on the following result which can be located in [9].

Lemma 2 Suppose that $\rho_{1}(z)$ is analytic in $U$ and $\rho_{2}(z)$ is convex univalent in $U$ with $\rho_{1}(0)=\rho_{2}(0)$. If

$$
\rho_{1}(z)+\frac{1}{\kappa}\left(\rho_{1}^{\prime}(z)\right) \prec \rho_{2}(z)
$$

for a nonzero complex constant number $\kappa$ with $\Re(\kappa) \geq 0$, then $\rho_{1}(z) \prec \rho_{2}(z)$.

\section{Results}

In the result section, we present the sufficient condition for functions to be in the class $\Lambda l_{s}$.

Theorem 3 Let $f \in \Lambda$, and for some constants $s \in \mathbb{R} \backslash\{0\}$ define the functional

$$
\Psi_{s}(z)=\frac{1}{2 s}\left(\frac{z f^{\prime \prime}(z)}{f^{\prime}(z)}-\frac{z^{3}}{\frac{z f^{\prime}(z)}{f(z)}}\right) .
$$

Then $\Psi_{s}(z) \prec \Delta f(z)$ if one of the subordinations occurs:

- $1+s\left(z \Psi_{s}^{\prime}(z)\right) \prec(1+z)^{1 / 2}, s \geq \max \left\{\left|s_{0}\right|,\left|s_{1}\right|\right\}$, where

$$
s_{0}=\frac{(2(-1+\sqrt{2}+\log (2)-\log (1+\sqrt{2})))}{\left(3^{2 / 3} \Gamma(2 / 3) A_{l}(1)-1\right)}, \quad s_{1}=\frac{(2(\log (2)-1))}{\left(3^{2 / 3} \Gamma(2 / 3) A l(-1)+1\right)} ;
$$

- $1+s\left(z \frac{\Psi_{s}^{\prime}(z)}{\Psi_{s}(z)}\right) \prec(1+z)^{1 / 2}, s \geq \max \left\{\left|s_{2}\right|,\left|s_{3}\right|\right\}$, where for some $m \in \mathbb{N}$

$$
\begin{aligned}
& s_{2}=\frac{6(-1+\sqrt{2}+\log (2)-\log (1+\sqrt{2}))}{2 \log (3)+3 \log (\Gamma(2 / 3))+3 \log \left(A_{l}(1)\right)}, \\
& s_{3}=\frac{6(1-\log (2))}{\left|(6 m+3) \pi-i\left(\log (9)+3 \log \left(A_{l}(-1) \Gamma(2 / 3)\right)\right)\right|} ;
\end{aligned}
$$

- $1+s\left(z \frac{\Psi_{s}^{\prime}(z)}{\Psi_{s}^{2}(z)}\right) \prec(1+z)^{1 / 2}, s \geq \max \left\{\left|s_{4}\right|,\left|s_{5}\right|\right\}$, where

$$
s_{4}=\frac{113 \times 3^{2 / 3} \Gamma(2 / 3) A_{l}(1)}{250\left(3^{2 / 3} \Gamma(2 / 3) A_{l}(1)-1\right)}, \quad s_{5}=\frac{3 \times 3^{2 / 3} \Gamma(2 / 3) A_{l}(-1)}{5\left(-1-3^{2 / 3} \Gamma(2 / 3) A_{l}(-1)\right)} .
$$

Proof 1 Case I: $1+s\left(z \Psi_{s}^{\prime}(z)\right) \prec(1+z)^{1 / 2}$.

Define a function $F_{s}: U \rightarrow \mathbb{C}$ formulating by

$$
F_{s}(z)=1+\frac{2}{s}\left((1+z)^{1 / 2}-\log \left(1+(1+z)^{1 / 2}\right)+\log (2)-1\right) .
$$

Obviously, the analytic function $F_{s}(z)$ achieves $F_{s}(0)=1$ and satisfies

$$
1+s\left(z F_{s}^{\prime}(z)\right)=(1+z)^{1 / 2} .
$$

Thus, we obtain $\mathfrak{F}(z):=s\left(z F_{s}^{\prime}(z)\right)=(1+z)^{1 / 2}-1$ is starlike in $U$. Consequently, by Lemma 2 , it yields

$$
1+s\left(z \Psi_{s}^{\prime}(z)\right) \prec 1+s\left(z F_{s}^{\prime}(z)\right) \quad \Rightarrow \quad \Psi_{s}(z) \prec F_{s}(z) .
$$


To complete this argument, we must prove that $F_{s}(z) \prec \Delta f(z)$, or equivalently, $F_{s}(z) \prec$ $\mathbb{A}_{l}(z)$. Evidently, the function $F_{s}(z)$ is increasing in the interval $(-1,1)$, which fulfils the inequality $F_{s}(-1) \leq F_{s}(1)$. Since

$$
\begin{aligned}
-\frac{J_{-1 / 3}\left(\frac{2}{3}\right)+J_{1 / 3}\left(\frac{2}{3}\right)}{3} & \approx-\left(3^{2 / 3} \Gamma\left(\frac{2}{3}\right)\right) A_{l}(-1) \leq F_{s}(-1) \\
& \leq F_{s}(1) \leq 3^{2 / 3} \Gamma\left(\frac{2}{3}\right) A_{l}(1) \approx \frac{I_{-1 / 3}\left(\frac{2}{3}\right)-I_{1 / 3}\left(\frac{2}{3}\right)}{3},
\end{aligned}
$$

where $s \geq \max \left\{\left|s_{0}\right|,\left|s_{1}\right|\right\}$ such that

$$
s_{0}=\frac{(2(-1+\sqrt{2}+\log (2)-\log (1+\sqrt{2})))}{\left(3^{2 / 3} \Gamma(2 / 3) A_{l}(1)-1\right)}, \quad s_{1}=\frac{(2(\log (2)-1))}{\left(3^{2 / 3} \Gamma(2 / 3) A_{l}(-1)+1\right)} ;
$$

then we obtain

$$
\Psi_{s}(z) \prec F_{s}(z) \prec \Delta f(z) \quad \Rightarrow \quad \Psi_{s}(z) \prec \Delta f(z) .
$$

This indicates that $f \in \Lambda t_{s}$.

Case II: $1+s\left(\frac{z \Psi_{s}^{\prime}(z)}{\Psi_{s}(z)}\right) \prec(1+z)^{1 / 2}$. Construct the function $\Omega_{s}: U \rightarrow \mathbb{C}$ as follows:

$$
\Omega_{s}(z)=\exp \left(\frac{2}{s}\left((1+z)^{1 / 2}-\log \left(1+(1+z)^{1 / 2}\right)+\log (2)-1\right)\right) .
$$

The function $\Omega_{s}(z)$ is analytic in $U$ having $\Omega_{s}(0)=1$, and it is a solution of the differential equation

$$
1+s\left(\frac{z \Omega_{s}^{\prime}(z)}{\Omega_{s}(z)}\right)=(1+z)^{1 / 2}, \quad z \in U
$$

By considering $\mathfrak{F}(z)=(1+z)^{1 / 2}-1$, which is starlike in $U$ and $\mathfrak{G}(z)=\mathfrak{F}(z)+1$, we have

$$
\Re\left(\frac{z \mathfrak{F}^{\prime}(z)}{\mathfrak{F}(z)}\right)=\Re\left(\frac{z \mathfrak{G}^{\prime}(z)}{\mathfrak{F}(z)}\right)>0
$$

Again by Lemma 2, we have

$$
1+s\left(\frac{z \Psi_{s}^{\prime}(z)}{\Psi_{s}(z)}\right) \prec 1+s\left(\frac{z \Omega_{s}^{\prime}(z)}{\Omega_{s}(z)}\right) \Rightarrow \Psi_{s}(z) \prec \Omega_{s}(z) .
$$

Consequently, we get

$$
\begin{aligned}
-\frac{J_{-1 / 3}\left(\frac{2}{3}\right)+J_{1 / 3}\left(\frac{2}{3}\right)}{3} & \approx-\left(3^{2 / 3} \Gamma\left(\frac{2}{3}\right)\right) A l(-1) \leq \Omega_{s}(-1) \\
& \leq \Omega_{s}(1) \leq\left(3^{2 / 3} \Gamma\left(\frac{2}{3}\right) A_{l}(-1) \approx \frac{I_{-1 / 3}\left(\frac{2}{3}\right)-I_{1 / 3}\left(\frac{2}{3}\right)}{3},\right.
\end{aligned}
$$


whenever $s \geq \max \left\{\left|s_{2}\right|,\left|s_{3}\right|\right\}$, where

$$
\begin{aligned}
& s_{2}=\frac{6(-1+\sqrt{2}+\log (2)-\log (1+\sqrt{2}))}{2 \log (3)+3 \log (\Gamma(2 / 3))+3 \log (A l(1))}, \\
& s_{3}=\frac{6(1-\log (2))}{\left|(6 m+3) \pi-i\left(\log (9)+3 \log \left(A_{l}(-1) \Gamma(2 / 3)\right)\right)\right|} .
\end{aligned}
$$

This indicates that the subordination inequalities

$$
\Psi_{s}(z) \prec \Omega_{s}(z) \prec \Delta f(z) \quad \Rightarrow \quad \Psi_{s}(z) \prec \Delta f(z) .
$$

Hence, $f \in \Lambda l_{s}$.

Case III: $1+s\left(\frac{z \Psi_{s}^{\prime}(z)}{\Psi_{s}^{2}(z)}\right) \prec(1+z)^{1 / 2}$. Consider the function $\partial_{s}: U \rightarrow \mathbb{C}$ by

$$
\partial_{s}(z)=\frac{1}{\left(1-\frac{2}{s}\left((1+z)^{1 / 2}-\log \left(1+(1+z)^{1 / 2}\right) \log (2)-1\right)\right)} .
$$

Clearly, $\partial_{s}(z)$ is analytic in $U$ such that $\partial_{\varepsilon}(0)=1$, and it satisfies

$$
1+s\left(\frac{z \varlimsup_{s}^{\prime}(z)}{\varlimsup_{s}(z)}\right)=(1+z)^{1 / 2} .
$$

By Lemma 2, one can have

$$
1+s\left(\frac{z \Psi_{s}^{\prime}(z)}{\Psi_{s}^{2}(z)}\right) \prec 1+s\left(\frac{z \varlimsup_{s}^{\prime}(z)}{\partial_{s}^{2}(z)}\right) \Rightarrow \Psi_{s}(z) \prec \partial_{s}(z) .
$$

This implies

$$
\begin{aligned}
-\frac{J_{-1 / 3}\left(\frac{2}{3}\right)+J_{1 / 3}\left(\frac{2}{3}\right)}{3} & \approx-\left(3^{2 / 3} \Gamma\left(\frac{2}{3}\right)\right) A_{l}(-1) \leq \mathrm{Ј}_{s}(-1) \\
& \leq \partial_{s}(1) \leq 3^{2 / 3} \Gamma\left(\frac{2}{3}\right) A_{l}(1) \approx \frac{I_{-1 / 3}\left(\frac{2}{3}\right)-I_{1 / 3}\left(\frac{2}{3}\right)}{3},
\end{aligned}
$$

whenever $s \geq \max \left\{\left|s_{4}\right|,\left|s_{5}\right|\right\}$, where

$$
s_{4}=\frac{113 \times 3^{2 / 3} \Gamma(2 / 3) A_{l}(1)}{250\left(3^{2 / 3} \Gamma(2 / 3) A_{l}(1)-1\right)}, \quad s_{5}=\frac{3 \times 3^{2 / 3} \Gamma(2 / 3) A_{l}(-1)}{5\left(-1-3^{2 / 3} \Gamma(2 / 3) A l(-1)\right)} .
$$

As a conclusion, we have the consequences

$$
\Psi_{s}(z) \prec \partial_{s}(z) \prec \Delta f(z) \quad \Rightarrow \quad \Psi_{s}(z) \prec \Delta f(z) .
$$

This leads to $f \in \Lambda l_{s}$.

Other results are given in the next theorem.

Theorem 4 Let $f \in \Lambda$ and

$$
\Psi_{s}(z)=\frac{1}{2 s}\left(\frac{z f^{\prime \prime}(z)}{f^{\prime}(z)}-\frac{z^{3}}{\frac{z f^{\prime}(z)}{f(z)}}\right) .
$$


If

- $\Psi_{s}(z) \ll \Delta f(z)$ and $f \in \mathcal{C}$ (the class of convex analytic functions in $U$ ), then $f \in \Lambda s_{s}$ for $|z| \in(0.28, \sqrt{2}-1]$;

- $\Psi_{s}(z) \ll \Delta f(z)$ and $f \in \mathcal{S}^{*}$ (the class of starlike analytic functions in $U$ ), then $f \in \Lambda l_{s}$ for $|z| \in(0.21,0.3)$;

- $f \in \Lambda l_{s}$ and $f \in U_{\wp}, \wp \geq 1.65$ (the set of all locally univalent functions of order $\wp$ ), then

$$
\Psi_{s}^{\prime}(z) \ll(\Delta f(z))^{\prime}, \quad|z| \leq(\wp+1)-\sqrt{\wp^{2}+2 \wp} .
$$

Proof 2 For the first conclusion, since $f$ is convex and $A_{l}$ is convex in $U$ whenever $\Re(z)>0$, then $\Delta f \in \mathcal{C}$ (see [12]). By [10, Corollary 1], we have $\Psi_{s}(z) \prec \Delta f(z)$, and hence $f \in \Lambda s_{s}$ for $|z| \in(0.28, \sqrt{2}-1]$. The second part comes from the fact that $\Delta f \in \mathcal{S}^{*}$, and hence by [10, Corollary 2] we get $\Psi_{s}(z) \prec \Delta f(z) \Rightarrow f \in \Lambda_{s}$ for $|z| \in(0.21,0.3)$. Lastly, in view of [10, Theorem 3], we have the desired assertion.

\title{
4 Conclusion
}

From the above study, we formulated a new linear operator utilizing the Airy function. By using the new operator, we defined a new class of analytic functions and investigated its properties. We showed that the operator can be approximated by well-known special functions. Sufficient conditions are studied to be sure that the normalized function $f$ is recognized in the new class. For future works, one can suggest new classes of analytic functions involving the linear operator.

\author{
Acknowledgements \\ The authors would like to express their full thanks to the respected editor, editorial office, and reviewers for the in-depth \\ advise, which improved our paper. \\ Funding \\ Not applicable. \\ Availability of data and materials \\ Not applicable. \\ Competing interests \\ The authors declare that they have no competing interests. \\ Authors' contributions \\ All authors contributed equally and significantly to writing this article. All authors read and agreed to the published \\ version of the manuscript. \\ Author details \\ 'IEEE: 94086547, Kuala Lumpur, 59200, Malaysia. ²Department of Mathematics, Cankaya University, 06530 Balgat, Ankara, \\ Turkey. ${ }^{3}$ Institute of Space Sciences, R76900 Magurele-Bucharest, Romania. ${ }^{4}$ Department of Medical Research, China \\ Medical University, Taichung 40402, Taiwan.
}

\section{Publisher's Note}

Springer Nature remains neutral with regard to jurisdictional claims in published maps and institutional affiliations.

Received: 6 June 2021 Accepted: 22 July 2021 Published online: 04 August 2021

References

1. Carlson, B.C., Shaffer, D.B.: Starlike and prestarlike hypergeometric functions. SIAM J. Math. Anal. 15(4), $737-745$ (1984)

2. Owa, S., Srivastava, H.M.: Univalent and starlike generalized hypergeometric functions. Can. J. Math. 39(5), 1057-1077 (1987)

3. Ibrahim, R.W., Darus, M.: On analytic functions associated with the Dziok-Srivastava linear operator and Srivastava-Owa fractional integral operator. Arab. J. Sci. Eng. 36(3), 441-450 (2011)

4. Ibrahim, R.W., Darus, M.: New classes of analytic functions involving generalized Noor integral operator. J. Inequal. Appl. 2008, Article ID 390435 (2008) 
5. Airy, G.B.: On the intensity of light in the neighbourhood of a caustic. Trans. Camb. Philos. Soc. 6, 379 (1838)

6. Baik, J., Liechty, K., Schehr, G.: On the joint distribution of the maximum and its position of the Airy2 process minus a parabola. J. Math. Phys. 53(8), 083303 (2012)

7. Abou Jaoude, A.: The paradigm of complex probability and Claude Shannon's information theory. Syst. Sci. Control Eng. 5(1), 380-425 (2017)

8. Ibrahim, R.W., Darus, M.: Analytic study of complex fractional Tsallis' entropy with applications in CNNs. Entropy 20(10), $722(2018)$

9. Miller, S.S., Mocanu, P.T.: Differential Subordinations: Theory and Applications. CRC Press, Boca Raton (2000)

10. Campbell, D.M.: Majorization-subordination theorems for locally univalent functions, II. Can. J. Math. 25(2), 420-425 (1973)

11. MacGregor, T.H.: Majorization by univalent functions. Duke Math. J. 34(1), 95-102 (1967)

12. Ruscheweyh, S.: Convolutions in Geometric Function Theory. University of Montreal Press, Montreal (1982)

\section{Submit your manuscript to a SpringerOpen ${ }^{\circ}$} journal and benefit from:

- Convenient online submission

- Rigorous peer review

- Open access: articles freely available online

- High visibility within the field

- Retaining the copyright to your article

Submit your next manuscript at $\gg$ springeropen.com 